\title{
Etude de l'ablation d'une cible graphite par un laser U.V., application à la réalisation de couches de carbone dur
}

\author{
C. Germain, C. Girault, J. Aubreton, A. Catherinot, S. Bec* et A. Tonck* \\ Laboratoire Céramiques Nouvelles, "Plasma-Laser-Matériaux", Faculté des Sciences, \\ 123 avenue A. Thomas, 87060 Limoges, France \\ * Laboratoire de Tribologie et Dynamique des Systèmes, URA 855 du CNRS, Ecole \\ Centrale de Lyon, BP. 163, 69131 Ecully, France
}

\begin{abstract}
Résumé : Le panache plasma cré lors de l'interaction entre le faisceau d'un laser U.V. et une cible de carbone graphite a été étudié par trois techniques d'analyse : la spectroscopie optique d'émission, la détection de charges et la double sonde de Langmuir. La comparaison des vitesses des ions obtenues par un détecteur d'ions et par spectroscopie nous permet de faire une hypothèse sur la structure du panache plasma. Puis, nous donnons quelques résultats sur la réalisation de couches minces de carbone dur sur divers types de substrats.
\end{abstract}

Nous avons utilisé la technique d'ablation laser dans le but d'obtenir des couches carbone possédant des propriétés voisines de celles du diamant (grande dureté, résistivité électrique et conductivité thermique, transparence optique, inertie chimique...). Le principe consiste à pulvériser une cible graphite avec un laser impulsionnel et à recueillir la matière éjectée sur un substrat. Dans le but de maîtriser les étapes intervenant lors du dépôt $\mathrm{du}$ film, il était nécessaire de connaître et comprendre les phénomènes mis en jeu lors du transport des espèces dans le panache plasma de la cible vers le substrat. Pour celà, nous avons utilisé deux moyens d'analyses : la spectroscopie optique d'émission et la détection de charges.

La faible divergence $(<0,15 \mathrm{mrad})$ du faisceau laser (laser U.V. KrF, $\lambda=248 \mathrm{~nm}$ ) permet d'atteindre des fluences laser au niveau de la cible très importantes $(>2000$ $\mathrm{J} / \mathrm{cm}^{2}$ ). Les expériences sont réalisées dans une enceinte ultra-vide (vide limite \# $10^{-5} \mathrm{~Pa}$ ). Une analyse du panache plasma par spectroscopie optique d'émission résolue spatialement et temporellement $(1,2)$ en fonction de la fluence, a permis d'observer la présence des ions $\mathrm{C}^{+*}$ et $\mathrm{C}^{++*}$. Nous avons donc cherché à déterminer leur vitesse par deux méthodes : par spectroscopie optique d'émission et par détection de charges. Les résultats obtenus $(2)$ montrent que, dans nos conditions expérimentales $\left(\mathrm{F}>10 \mathrm{~J} / \mathrm{cm}^{2}\right)$, les vitesses déduites du temps de vol des ions à $400 \mathrm{~mm}$ de la surface de la cible sont similaires à celles des premiers ions observés par spectroscopie optique d'émission. Le signal électrique recueilli sur le collecteur semble donc correspondre 
seulement aux premiers ions du panache plasma. Ceci nous amène à formuler l'hypothèse suivante sur sa structure : dès les premiers instants de l'interaction, dès photoélectrons sont émis ; ils vont créer un champ électrique intense qui va "tirer" hors du panache plasma des ions (ce sont eux que l'on détectera). Derrière, le panache est. constitué d'un plasma globalement neutre géré par diffusion ambipolaire, où les ions et les électrons sont appariés, ce qui explique que l'on ne peut pas les différencier lorsqu'ils sont recueillis par le collecteur.

$\mathrm{Si}$ on regarde l'énergie des premiers ions, elle peut dépasser $1 \mathrm{keV}$ lorsque la fluence est élevée $\left(>2000 \mathrm{~J} / \mathrm{cm}^{2}\right)\left({ }^{2}\right)$, ce qui aura forcément des conséquences sur la formation d'un dépôt.

Les couches minces sont réalisées dans les conditions suivantes :

- température inférieure ou égale à $100{ }^{\circ} \mathrm{C}$ (pourcentage $\mathrm{sp}^{3} / \mathrm{sp}^{2}$ maximum à température ambiante $\left({ }^{2}\right)$ ),

- distance cible-substrat $=30 \mathrm{~mm}$,

- fluence laser : 10 à $5000 \mathrm{~J} / \mathrm{cm}^{2}$, taux de répétition du laser de $10 \mathrm{~Hz}$, plexiglass...

- divers types de substrat : $\mathrm{Si}, \mathrm{ZnS}, \mathrm{Mo}$, acier, $\mathrm{Al}_{2} \mathrm{O}_{3}$, verre, téflon,

L'adhérence des films augmente lorsque la fluence et la température augmentent. A température ambiante, les films sont adhérents sur les substrats cités ci-dessus, hormis sur les pastilles de $\mathrm{ZnS}$ où il faut atteindre des températures de substrat de l'ordre de $100^{\circ} \mathrm{C}$ pour que le film ne se décolle pas. Les couches sont transparentes à plus de 90 \% aux rayonnements infra rouge. La rugosité de surface, mesurée par microscopie à force atomique à l'Université de Poitiers (U.R.A. 130 CNRS), est inférieure à $2,5 \mathrm{~nm}$. Une valeur de dureté de $80 \mathrm{GPa}$ ainsi qu'une très grande élasticité (module d'Young supérieure à $1000 \mathrm{GPa}$ ) a été mesurée par nanoindentation à l'Ecole Centrale de Lyon, ces valeurs étant à confïmer par la suite. Elles sont comparables à celle du diamant (dureté : $100-120 \mathrm{GPa}$ et module d'élasticité de l'ordre de $1200 \mathrm{GPa}$ ) et à celles trouvées par C. B. COLLINS et al. $(80-100 \mathrm{GPa})$ qui réalisent un matériau appelé "diamant amorphe" par ablation d'une cible graphite avec un laser YAG $(3)$.

L'étude du panache plasma créé lors de l'interaction entre le laser U.V. et la cible graphite nous a permis de mieux comprendre la structure de la plume plasma et les phénomènes mis en jeu lors de la réalisation du dépôt.

Les films obtenus de par leur propriétés (grande dureté et élasticité, transparence optique, adhérence sur différents substrats) et le fait qu'ils sont réalisés à température ambiante, leur confere un domaine d'application étendu.

\section{Références :}

[1] C. GERMAIN, C. GIRAULT, J. AUBRETON and A. CATHERINOT, Appl. Surf. Sci. 69 (1993) 359.

[2] C. GERMAIN, C. GIRAULT, R. GISBERT, J. AUBRETON and A. CATHERINOT, Diamond and Related Materials, 3 (1994) 598.

[3] C. B. COLLINS, F. DAVANLOO, T. J. LEE, J. H. YOU and H. PARK, Mat. Res. Symp. Proc. vol. 285 (1993) 548. 